\title{
Duchenne muscular dystrophy in the Western Cape, South Africa: Where do we come from and where are we going?
}

\author{
A I Esterhuizen, ${ }^{1,2} \mathrm{MSc}$ (Med); L J Greenberg, ${ }^{1} \mathrm{PhD}$; R Ballo, ${ }^{1,3} \mathrm{PhD}$; R G Goliath, ${ }^{1,2} \mathrm{PhD}$; \\ J M Wilmshurst, ${ }^{1,4}$ MBBS, MRCP (Lond), FCPaed (SA), MD \\ ${ }^{1}$ Division of Human Genetics, Department of Pathology, Institute of Infectious Diseases and Molecular Medicine, University of Cape Town, \\ South Africa \\ ${ }^{2}$ National Health Laboratory Service, Groote Schuur Hospital, Cape Town, South Africa \\ ${ }^{3}$ Division of Cell Biology, Department of Human Biology, Faculty of Medicine, University of Cape Town, South Africa \\ ${ }^{4}$ Department of Paediatric Neurology and Neurophysiology, Red Cross War Memorial Children's Hospital, University of Cape Town, South Africa
}

Corresponding author: A I Esterhuizen (alina.esterhuizen@uct.ac.za)

Duchenne muscular dystrophy (DMD) is one of the most common and severe of the inherited dystrophies, with an incidence of 1 in 3500 live, male births worldwide. Becker muscular dystrophy (BMD) has a lower incidence of 1:14 000 - 18000 boys and a milder progression and longer life expectancy. Over the last two decades, better understanding of the underlying disease aetiology as well as major advances in medical technology have brought about significantly improved genetic diagnosis and clinical care for B/DMD patients. Exciting developments in the field of gene-based therapies have once again put B/DMD in the limelight, with renewed focus on the importance of comprehensive genetic testing protocols. We present a historical overview of the medical and molecular service for B/DMD offered over the last three decades in South Africa, specifically in the Western Cape, from a clinical as well as a laboratory perspective.

S Afr Med J 2016;106(6 Suppl 1):S67-S71. DOI:10.7196/SAMJ.2016.v106i6.10999

Duchenne muscular dystrophy (DMD) is one of the most common and severe of the inherited dystrophies, with an incidence of 1 in 3500 live, male births worldwide. Long before the discovery of the $D M D$ gene, early investigators noted the $\mathrm{X}$-linked recessive heritability of DMD by scrutiny of affected family pedigrees. ${ }^{[1]}$ The allelic form of DMD, Becker muscular dystrophy (BMD), has a lower incidence of 1:14 000 - 18000 boys, a milder progression and longer life expectancy. ${ }^{[2]}$

Over the last two decades, better understanding of the underlying disease aetiology as well as major advances in medical technology have brought about significantly improved genetic diagnosis and clinical care for B/DMD patients. However, a cure, while in sight, remains elusive. This said, exciting developments in the field of genebased therapies have once again put B/DMD in the limelight, with renewed focus on the importance of comprehensive genetic testing protocols. We present a historical overview of the medical service for B/DMD in South Africa (SA), specifically in the Western Cape, from a clinical as well as a genetic perspective.

\section{Clinical description}

DMD manifests in boys between 2 and 5 years of age, with delayed motor milestones and symptoms such as frequent falling, difficulty in getting up, gait problems and toe walking. Pseudohypertrophic calves, lumbar lordosis and weakness of the neck flexors are typical. Weakness of the knee and hip extensors results in the Gower's manoeuvre, where the child uses his hands and arms to 'climb' up his body to rise from a prone position. Muscle weakness is progressive, with loss of independent ambulation between 9 and 13 years of age for DMD and $>16$ years for BMD patients. Previously, death before the age of 19 years from respiratory and cardiac complications was common, but current multidisciplinary care allows survival into adulthood. Rare cases have a very localised disease expression, e.g. cardiac disease. A degree of intellectual disability is seen in over a third of the affected children ${ }^{[3]}$ BMD patients have a more diverse presentation and progression, in some cases showing only mild myalgia and muscle cramps with no weakness. ${ }^{[4]}$

\section{B/DMD genetics}

Localisation of the $D M D$ gene to Xp21 was achieved by cytogenetic studies of DMD-manifesting females carrying $\mathrm{X}$-autosome translocations and extensive work with positional cloning. ${ }^{[5]}$ Full characterisation of the gene revealed $2.4 \mathrm{Mb}$ of genomic sequence and a 14kb mRNA transcript, making it one of the largest known human genes, comprising 79 exons, at least 8 tissue-specific promoters and 2 polyA-addition sites. Owing to its large size, the $D M D$ gene is prone to mutations, of which approximately $70 \%$ are exonic deletions and duplications. These tend to cluster within two hot-spot regions spanning exons 44 - 53 and exons 2 - 20. The remaining $25-35 \%$ of the disease-associated changes are small/point alterations, mostly nonsense, frame-shift or splice site mutations. ${ }^{[6]}$ These do not appear to exhibit any clustering effect and because of the large size of the gene, continue to present a diagnostic challenge.

A full-length dystrophin is a large, rod-like cytoskeletal protein localised to the inner surface of muscle fibres, bridging the inner cytoskeleton (F-actin) and the extracellular matrix, providing structural integrity to the muscle cell membrane. ${ }^{[]]}$Two main factors appear to influence severity of the B/DMD phenotype: localisation of the causative mutation within the gene relative to the dystrophin critical functional domains; and the disruption/maintenance of the translational open reading frame (ORF). The 'reading frame hypothesis' which fits $\sim 90 \%$ of DMD cases, argues that ORFdisrupting mutations cause premature termination of translation and nonsense-mediated decay (NMD), resulting in complete absence of dystrophin and a more severe phenotype. Mutations affecting the actin-binding $\mathrm{N}$-terminus, the carboxy-terminus and the cysteinerich regions of dystrophin generally cause a severe phenotype, even if the rearrangement is in-frame. The central and distal rod domains, however, appear to be almost dispensable and cases of in-frame 


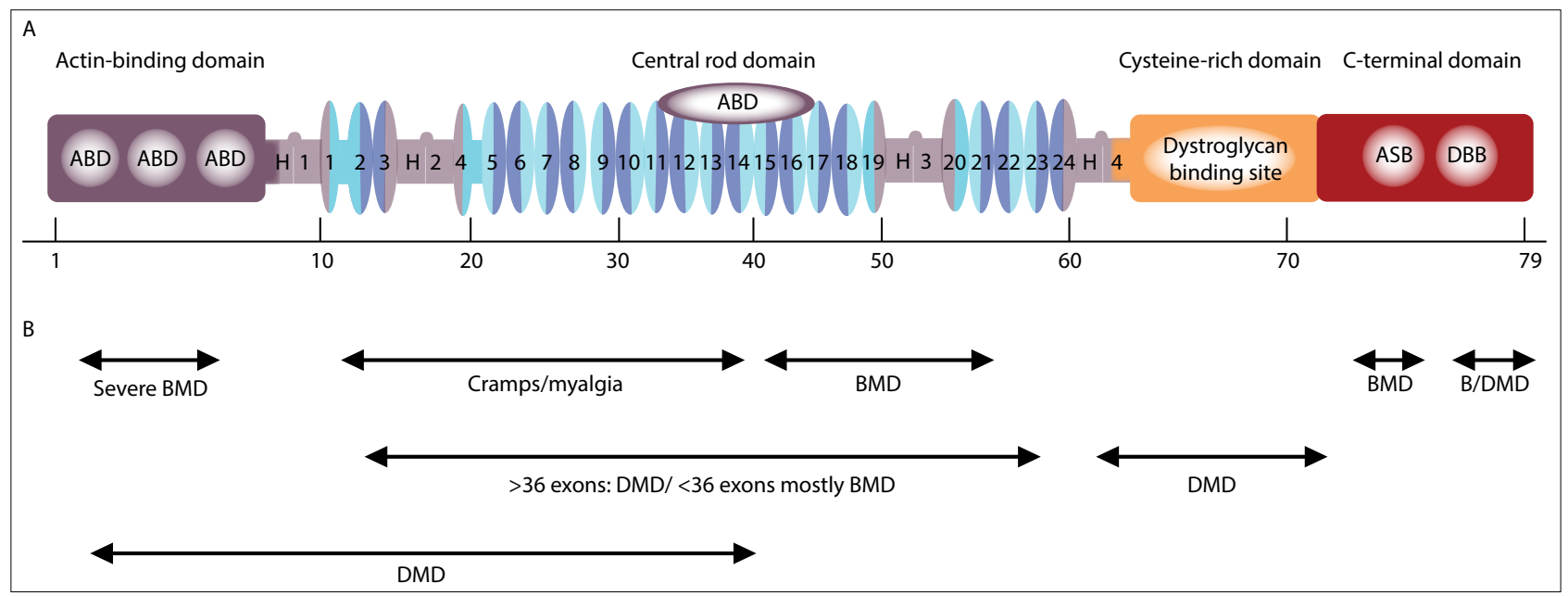

Fig. 1. Correlation between the location of in-frame deletions and the BMD or DMD phenotype. (A) Schematic drawing of the dystrophin protein with its functional domains. The location of the different exons is shown underneath the protein. (B) Graphic overview of the relation between the location of in-frame deletions and the severity of the phenotype (adapted from Aartsma-Rus, et al. ${ }^{[6]}$ ).

deletions of approximately half of the gene have been described, with only a mild effect on the phenotype ${ }^{[8]}$ However, phenotypes can vary between patients carrying identical mutations even within one family, which could be a result of the mutation's epigenetic context. ${ }^{[6]}$ The schematic overview in Fig. 1 reflects general findings for in-frame deletions.

\section{B/DMD in South Africa: The early days}

The clinical database and DNA registry in the Division of Human Genetics at the University of Cape Town (UCT), were the first undertakings of a genetic service for B/DMD in SA. This was supported by the neuromuscular clinic at Red Cross War Memorial Children's Hospital (RCWMCH) and numerous genetic outreach clinics spearheaded by Prof. Peter Beighton.

In 1987, Prof. Beighton and his team of clinicians and scientists set out to determine the feasibility of implementing a genetic testing service for B/DMD. Southern blot (SB) analysis using DNA probes targeting the $D M D$ locus became the first molecular genetic test offered in SA. SB remains the gold standard methodology for a number of genetic disorders, but it is a laborious and expensive technique with an extended turn-around time, as well as health and safety risks relating to the use of radioactivity. This analytical approach to B/DMD changed in the early 1990s when the polymerase chain reaction (PCR) launched molecular biology into a new era. The deletion hot-spots within the DMD gene could now be screened using multiplex PCR (mPCR) and agarose gel electrophoresis. Identification of the causative deletion in a proband confirmed the diagnosis and provided genetic closure to the family, enabling effective prenatal analysis and genetic counselling for future reproductive choice. The shortcoming of the mPCR, however, was its inability to detect duplications or deletions outside of the hot-spots, nor was it capable of detecting deletions in carrier females, due to the contribution from the normal X chromosome. The female B/DMD carrier status was still determined by restriction enzyme fragment length polymorphism analysis. In due course, this was replaced by microsatellite linkage studies with fluorescent PCR and capillary gel electrophoresis. In the absence of an identifiable mutation, however, the 5\% uncertainty linked to meiotic recombination even today presents a reporting and genetic counselling challenge.
During the period of 1987 - 1992, exonic deletions were identified in $50 \%$ of Indian, white and coloured patients. ${ }^{\left[{ }^{[9]}\right.}$ In contrast, only $22 \%$ of black patients had identifiable deletions. This was thought to suggest that unique mutations, not detectable by methods used at the time, might be more frequent in the black patients compared with other populations. It was also thought to explain the overall low B/ DMD prevalence in SA (1:40 000), compared with that in the UK. ${ }^{[9]}$ The increased DMD frequency in the Indian patients was found to be in line with findings reported from the UK. This observation prevails, though information relating to mutation prevalences in the SA population groups is not easily gathered from the diagnostic test requests, as the patients' ethnicity is generally not provided.

\section{The service today}

Genetic testing for B/DMD in the SA public sector is currently performed within the National Health Laboratory Service (NHLS) in Cape Town (Groote Schuur Hospital) and Johannesbsurg, and is extended to the State as well as private patients in and outside of SA. The mPCR method has since been replaced by the multiple ligasedependent probe amplification (MLPA) assay, though microsatellite linkage analysis is still available for families with no identified mutations. Introduction of the MLPA in 2007 was a significant improvement to the service, not only because of its coverage of all the exons within the $D M D$ gene, but also due to its dosage component, which enables identification of duplications in males and determines B/DMD carrier status in females (Fig. 2).

Since its inception in 1987, over 790 B/DMD families and 2000 individuals have been referred for testing to the diagnostic genetic service in Cape Town. Of those, 1264 individuals were referred for linkage analysis, as the only testing methodology available in the early days (these figures therefore include a number of unaffected family members). Implementation of the MLPA in 2007 significantly improved mutation detection rate from $~ 30 \%$ to $45 \%$. A similar pick-up rate has been reported in both Cape Town and Johannesburg laboratories, with a similar distribution of deletions $(\sim 36 \%)$ and duplications (9\%). ${ }^{[10]}$ This could be viewed as relatively low, compared with the internationally published mutation frequencies. ${ }^{[6]}$ However, it must be borne in mind that test requests are received from across southern Africa, often from regions with limited access to specialised diagnostic tools. Therefore, in addition to those patients who carry mutations other than exonic 


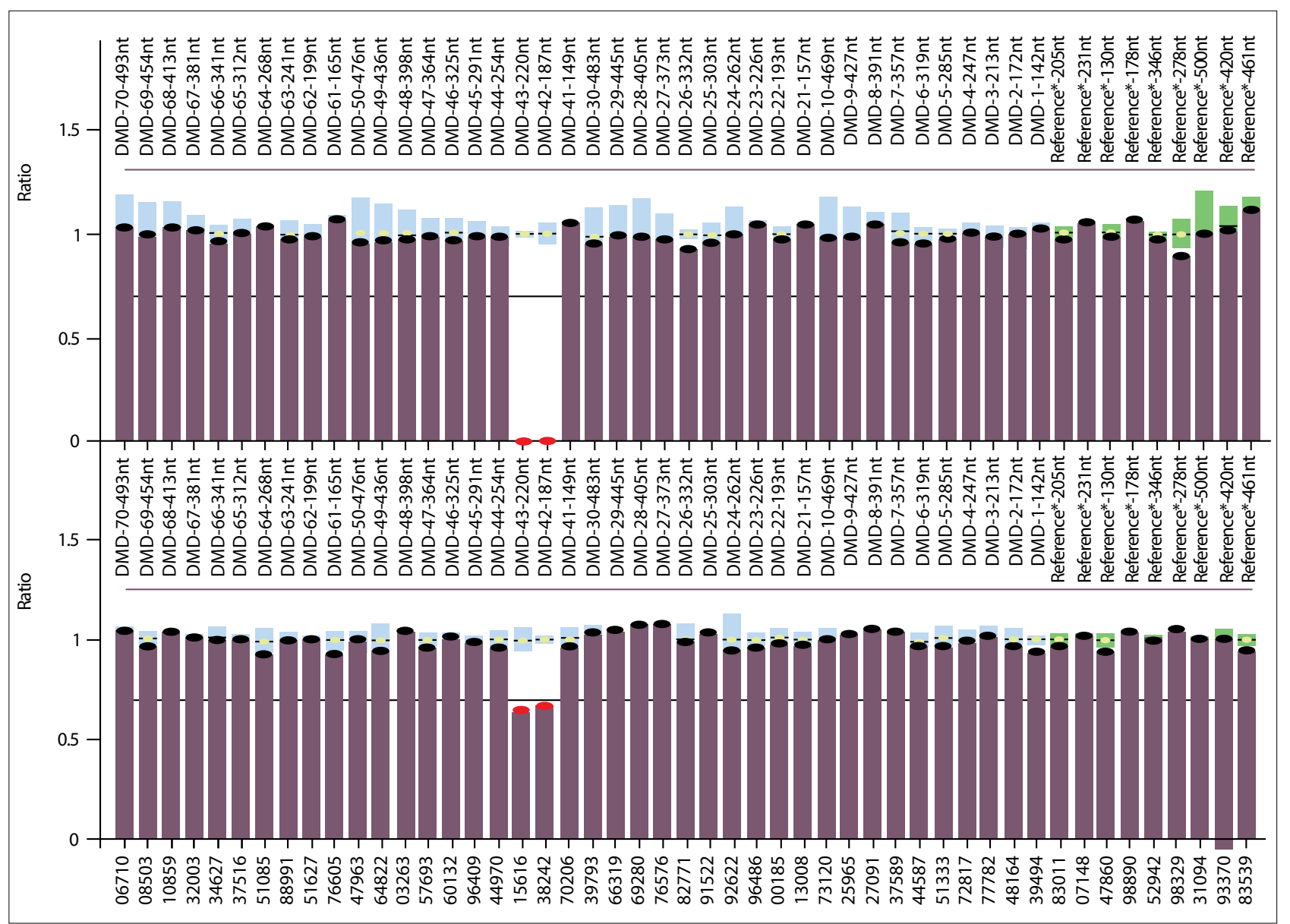

Fig. 2. MLPA dosage analysis of a DMD-affected boy with a deletion of exons 42 and 43 (top diagram) and his DMD-carrier mother (dosage ratio <0.6) using the Coffalyser.NET software (MRC-Holland, the Netherlands).

rearrangements (which are not detected with the MLPA), a number may require a clinical reassessment. Interestingly though, only about $50 \%$ of the definitively diagnosed B/DMD patients attending the dedicated neuromuscular clinic at RCWMCH carry exonic rearrangements, which is lower than expected and bears further investigation.

Because of its large size, testing for small/point mutations in the $D M D$ gene is challenging and has not yet become part of the routine diagnostic repertoire of the NHLS laboratories. Highresolution melting curve analysis (HRM) for small/point mutation detection was successfully attempted locally and the results were published by Esterhuizen et al. ${ }^{[1]}$ However, HRM in B/DMD has been superseded by next-generation sequencing (NGS ${ }^{[12]}$ which is currently undergoing validation in the Division of Human Genetics at UCT and the NHLS in Cape Town. Fig. 3 depicts the ideal workflow for a laboratory diagnosis of B/DMD, ultimately aiming at determining eligibility for gene-based therapy. RNA-based mutation analysis is not incorporated into the current laboratory protocols due to its limited benefit at this time. However, future availability of gene-based therapies may change this, as the downstream effects of a mutation are better discerned from muscle RNA.

\section{Clinical practice}

A dedicated neuromuscular service has been in existence at the RCWMCH for $>20$ years. Referrals are predominantly from the Western Cape, but children from further afield, including other African countries, are also seen. $\mathrm{B} / \mathrm{DMD}$ is the predominant neuromuscular condition managed, with $>90 \mathrm{~B} / \mathrm{DMD}$ children seen to date. In keeping with international protocols, the diagnosis in clinically suspected cases is confirmed by DNA analysis and if negative, by immunohistochemical (IHC) staining of muscle biopsies, unless a genetic diagnosis was previously made in a family member or if the child is medically unstable. ${ }^{[13]}$ Clinic visits are scheduled every $3-6$ months, where the families have access to genetic counselling and support from the Muscular Dystrophy Foundation of SA (MDFSA). ${ }^{[14]}$

After result delivery and genetic counselling, patients are managed according to the international guidelines within the capacity of the service facilities. ${ }^{[3,13]}$ A resident physiotherapist liaises with the ancillary support in the community, special schools and private therapists regarding interventions such as stretches, chest physiotherapy and orthotic devices (e.g. nocturnal ankle foot orthoses and wheelchairs). General health and power scores are serially recorded, including the presence of Gower's manoeuvre and evidence of contracture formation.

All children undergo regular cardiac assessments and receive enalapril or an equivalent agent as prophylaxis. Basic respiratory screens are performed at each clinic visit, and the patients and carers are educated with regard to good respiratory care and the use of devices such as the cough-assist. Corticosteroid intervention is commenced as soon as motor function appears affected (usually by 4 years of age). As corticosteroid side-effects include immunosuppression, prior to treatment children are vaccinated against varicella (Varilrix; GlaxoSmithKline, Belgium) and undergo testing to exclude tuberculosis. Subsequent monitoring for 


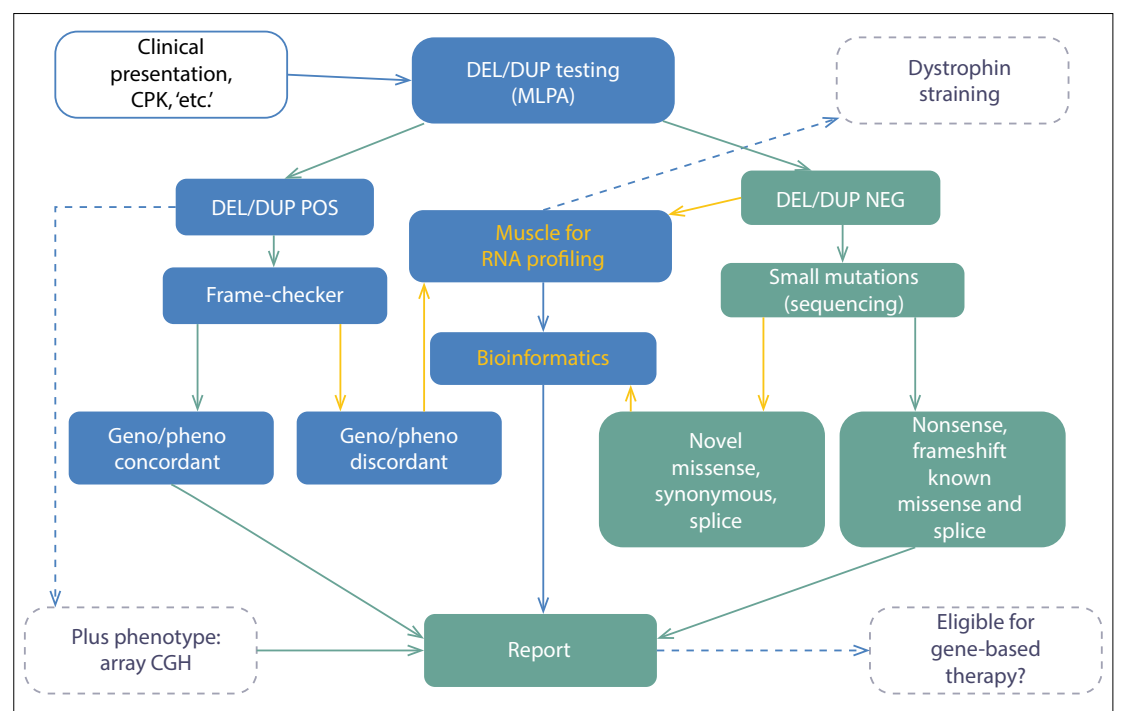

Fig. 3. A diagrammatic representation of a workflow for a genetic diagnosis and mutation characterisation in B/DMD. Blue fields: positive MLPA findings (exonic deletions or duplications) are analysed for their effect on the ORF (frame-checker), followed by genotype-phenotype correlation, e.g. interruption of the ORF should result in a more severe phenotype, etc. If this is concordant, a report can be issued. In discordant cases, a muscle biopsy for RNA testing and further bioinformatics analyses may be pursued before issuing a final result and determining eligibility for gene-based therapy. Green fields: Patients with negative MLPA results (no deletions or duplications) should be tested for small/point mutations using DNA sequencing (NGS and/or Sanger). Based on the outcome, a report may be issued or further bioinformatics analysis may be indicated. RNA testing of muscle biopsy material could be pursued directly, which will also detect intronic variants, i.e. pseudo-exon mutations. Further testing for copy number variations, e.g. array CGH, may be performed in patients with possible contiguous gene syndrome (plus phenotype). (CPK = creatine phosphokinase; $D E L / D U P=$ deletion/duplication; $C G H=$ comparative genomic hybridisation; GENO-PHENO = genotype-phenotype correlation.)

complications relating to chronic corticosteroid use includes annual bone mineral density assessments. Intercurrent infections are treated aggressively and patients are vaccinated against pneumococcus and influenza. Ambulatory devices for wheelchair-dependent children are reviewed at each clinic visit, and formal seating assessments are coordinated in specialist settings. Patients with scoliosis are referred to orthopaedic specialists. Bi-level positive airway pressure (BPAP) support for nocturnal hypoventilation is commenced following confirmation with polysomnography.

Schooling and education support runs in parallel with clinic care, which promotes placement of B/DMD patients in schools with appropriate facilities. As they mature, the children also need emotional support to deal with their illness. This is partially addressed through the service but a great deal of support is accessible to the patients and their families through (MDSFA). ${ }^{[14]}$

As a direct result of this multidisciplinary approach, B/DMD patients are surviving longer. While the significance of this extended lifespan cannot be discounted, the main aim is to ensure the best possible quality of life for the child and his (sometimes her) family. Many of the children demise through the course of their progressive disease, but an increasing number are growing beyond the capacity of a children's service to manage. The RCWMCH team are actively involved in interfacing with adult services and developing platforms for transition of care.

\section{Into the future \\ Genetic therapies}

The approaches to the development of genetic therapies can be roughly divided into two main fields: gene replacement and gene modification. Gene replacement strategies (viral vectors, utrophin up-regulation, myoblast and stem cell transplantation) aim at delivery of a normal copy of the gene directly to the affected tissue, either as naked DNA in a viral vector, or as a gene copy in a myoblast or stem cell. Proof-ofprinciple experiments on animal models (the mdx mouse) have been conducted and demonstrate that dystrophin expression can be restored to varying degrees to single muscles, muscle groups (limbs) or the entire body ${ }^{[15]}$ The major advantage of this strategy is that it can be applied to patients regardless of the underlying mutations. Although the research is showing promise, much work still needs to be done to overcome the mechanical and practical difficulties of gene delivery to the muscles, the immunological concerns and the high cost.

The other approach to genetic therapy, i.e. gene modification, also referred to as mutationor gene-based therapy, is currently receiving much attention. In terms of its application and delivery, this approach is viewed as less fraught with difficulties and significantly cheaper than gene replacement. Effectively, as identification of the particular mutation is the basis for this approach, 'personalised gene therapy' is becoming a reality.

At this time, closest to clinical application are the stop codon read-through and exon skipping therapies, which aim to restore the ORF and affect production of partially functional dystrophin, thereby ameliorating the severe Duchenne to a milder Becker phenotype. The stop codon readthrough agent, ataluren (Translarna; TC Therapeutics, USA), has recently received a conditional marketing authorisation from the European Medicines Agency (EMA) for use in ambulant Duchenne patients $>5$ years of age. PTC Therapeutics have also announced commencement of a rolling submission of a new drug application (NDA) to the US Food and Drug Administration (FDA). ${ }^{[16]}$ Similarly, intensive effort is made to demonstrate the safety and efficacy of exon skipping therapy for exon 51, which has shown considerable promise in phase II and III clinical trials. In October 2014, Prosensa Therapeutics announced that the FDA had given drisapersen (an exon skipping agent for exon 51) a fast track designation. ${ }^{[17-19]}$

\section{Registries and databases}

In 2010, the Division of Human Genetics at UCT obtained ethical approval for a formal repository entitled Genetics of Becker/ Duchenne Muscular Dystrophy: Biological Material Repository Including Database, by the UCT Faculty of Health Sciences Research Ethics Committee (HREC Ref. 234/2010). The repository holds all DNA material obtained through research and diagnostic testing for $\mathrm{B} / \mathrm{DMD}$, which may be needed for further work-up and/or B/DMD research.

In anticipation of a therapy-driven demand for the delineation of disease-causing genetic mutations, the neuromuscular service at RCWMCH, in collaboration with the Division of Human Genetics at UCT, is collating a database listing all patients with a clinical diagnosis of $\mathrm{B} / \mathrm{DMD}$ currently attending the neuromuscular clinic at RCWMCH (HREC Ref. 013/2013). The database holds information relating to each patient's clinical presentation, diagnosis, treatment, results of genetic testing, muscle biopsy sampling, etc., 
ultimately serving as a repository of information to identify DMD patients who may be suitable for a mutation-based intervention, as well as providing a source of information for future clinical trials, research and publication. The database currently holds information on 93 patients and is modelled on the TREAD-NMD database, an international care and site registry created to facilitate clinical research for neuromuscular diseases. ${ }^{[20,21]}$ Families are updated at each clinic visit of the stage of the data collection and how it relates to a potential gene therapy option. International liaison is also in place to ensure there is consistency in the patient selection as potential candidates for intervention.

\section{Research}

Traditionally, research into the genetics of B/DMD conducted at UCT/ RCWMCH has been focused on the translational aspects of mutation detection and diagnostic testing. Over the years, much of this research was funded by the MDFSA, whose collaborative ties with UCT go back to 1974 , when Prof. Peter Beighton was elected onto the first national MDFSA executive committee. This relationship has continued with various members of the Division of Human Genetics serving on the MDFSA committees and medical advisory boards.

Funded by the NHLS Research Trust, current genetic research at UCT/RCWMCH includes a project investigating dystrophin expression in human melanocytes to determine whether a less-invasive skin biopsy (and melanocyte culture) could replace muscle biopsy sampling for RNA-based confirmation and molecular diagnosis of B/DMD. ${ }^{[22]}$ Also underway is validation of NGS as a diagnostic methodology for small/point mutation detection in B/DMD.

From a clinical perspective, understanding the phenotypic (and genetic) profile of the SA patient population is important for a strategic and focused approach to care. Clinical studies have been conducted to assess the neurocognitive profile of the RCWMCH B/DMD patient cohort. ${ }^{[23]}$ Currently conducted at RCWMCH is a study analysing phenotypic data of all the B/DMD patients attending the RCWMCH muscle clinic, using multivariate regression and longitudinal analysis of variables ranging from the time of presentation, to cardiac and respiratory complications, to eventual loss of ambulation. This, in relation to the causative genetic defect, is aimed at better understanding of the genotype-phenotype correlations in local $\mathrm{B} / \mathrm{DMD}$ patients, as compared with the internationally published data. ${ }^{[4-6]}$ In due course, the database should be expanded to enable participation and data entry by clinicians from multiple centres, producing a more nationally representative $\mathrm{B} /$ DMD data repository and a potentially useful diagnostic/prognostic tool.

\section{Conclusion}

The $D M D$ gene was one of the first genes identified at the start of the molecular genetic era. The clinical and genetic service for B/DMD at UCT and RCWMCH was the first of its kind in SA and has evolved in line with international practice, even though it still incorporates the unique needs of children in SA. Today's clinicians and scientists continue to build on the foundations laid by the pioneering work of Prof. Beighton and his peers. The new era of genetic therapies and precision medicine presents exciting therapeutic options for patients and families, as well as areas of further research for scientists and clinicians. Thus, despite the challenges of a resource-limited setting, our research and data management efforts continue to strive towards better care and, one day soon, successful treatment, not just management, of B/DMD and its symptoms.

\section{References}

1. Emery AE. Duchenne muscular dystrophy--Meryon's disease. Neuromuscul Disord 1993;3(4):263266.

2. Koenig M, Beggs AH, Moyer M, et al. The molecular basis for Duchenne versus Becker muscular dystrophy: Correlation of severity with type of deletion. Am J Hum Genet 1989;45(4):498-506.

3. Bushby K, Finkel R, Birnkrant, et al. Diagnosis and management of Duchenne muscular dystrophy part 2: Implementation of multidisciplinary care. Lancet Neurol 2010;9(2):177-189. DOI:10.1016/ S1474-4422(09)70272-8

4. Beggs AH, Hoffman EP, Snyder JR, et al. Exploring the molecular basis for variability among patients Beggs AH, Hoffman EP, Snyder JR, et al. Exploring the molecular basis for variability among patients
with Becker muscular dystrophy: Dystrophin gene and protein studies. Am I Hum Genet 1991;49:54-

5. Koenig M, Hoffman EP, Bertelson CJ, Monaco AP, Feener C, Kunkel LM. Complete cloning of the duchenne muscular dystrophy (DMD) CDNA and preliminary genomic organization of the DMD gene in normal and affected individuals. Cell 1987;50(3):509-517.

6. Aartsma-Rus A, van Deutekom JCT, Fokkema IF, van Ommen GJB, Den Dunnen JT. Entries in the Leiden Duchenne muscular dystrophy mutation database: An overview of mutation type and paradoxical cases that confirm the reading-frame rule. Muscle Nerve 2006;34(2):135-144 DOI: $10.1002 /$ mus.20586

7. Blake DJ, Weir A, Newey SE, Davies KE. Function and genetics of dystrophin and dystrophin-related proteins in muscle. Physiol Rev 2002;82(2):291-329.

8. England SB, Nicholson LV, Johnson M, et al. Very mild muscular dystrophy associated with the deletion of 46\% of dystrophin. Nature 1990;343(6254):180-182.

Ballo R, Viljoen D, Beighton P. Duchenne and Becker muscular dystrophy prevalence in South Africa and molecular findings in 128 persons affected. S Afr Med J 1994;84(8 Pt 1):494-497.

10. Kerr R, Robinson C, Essop FB, Krause A. Genetic testing for Duchenne/Becker muscular dystrophy in Kerr R, Robinson C, Essop FB, Krause A. Genetic testing for Duchenne/Becker muscu/
Johannesburg, South Africa. S Afr Med J 2013;103(12):999. DOI:10.7196/samj.7274

11. Esterhuizen AI, Wilmshurst JM, Goliath RG, Greenberg LJ. Duchenne muscular dystrophy: Highresolution melting curve analysis as an affordable diagnostic mutation scanning tool in a South African cohort: Research. S Afr Med J 2014;104(11):779-784. DOI:10.7196/SAMJ.8257

12. Wang Y, Yang Y, Liu J, et al. Whole dystrophin gene analysis by next-generation sequencing: A comprehensive genetic diagnosis of Duchenne and Becker muscular dystrophy. Mol Genet Genomics 2014;289(5):1013-1021. DOI:10.1007/s00438-014-0847-z

13. Bushby K, Finkel R, Birnkrant DJ, et al. Diagnosis and management of Duchenne muscular dystrophy part 1: Diagnosis, and pharmacological and psychosocial management. Lancet Neurol 2010;9(1):7793. DOI:10.1016/S1474-4422(09)70271-6

14. Muscular Dystrophy Foundation of South Africa [Internet]. [cited 2016 May 6]. Available from: http:// www.mdsa.org.za

15. Fairclough RJ, Wood MJ, Davies KE. Therapy for Duchenne muscular dystrophy: renewed optimism from genetic approaches. Nat Rev Genet 2013;14(6):373-378. DOI:10.1038/nrg3460

16. Haas M, Vlcek V, Balabanov P, et al. European Medicines Agency review of ataluren for the treatment of ambulant patients aged 5 years and older with Duchenne muscular dystrophy resulting from a nonsense mutation in the dystrophin gene. Neuromuscul Disord 2015;25(1):5-13. DOI:10.1016/j. nmd.2014.11.011

7. Kole R, Krieg AM. Exon skipping therapy for Duchenne muscular dystrophy. Adv Drug Deliv Rev 2015;87:104-107. DOI:10.1016/j.addr.2015.05.008

8. Mendell JJR, Rodino-Klapac L, Rodino-Klapac LR, et al. Eteplirsen for the treatment of Duchenne muscular dystrophy. Ann Neurol 2013;74(5):637-47. DOI:10.1002/ana.23982

19. Voit T, Topaloglu H, Straub V. Safety and efficacy of drisapersen for the treatment of Duchenn muscular dystrophy (DEMAND II): An exploratory, randomised, placebo-controlled phase 2 study. muscular dystrophy (DEMAND II): An exploratory, randomised,

20. Bladen CL, Rafferty K, Straub V, et al. The TREAT-NMD Duchenne muscular dystrophy registries: Conception, design, and utilization by industry and academia. Hum Mutat 2013;34(11):1449-1457. DOI:10.1002/humu. 22390

21. Bladen CL, Salgado D, Monges S, et al. The TREAT-NMD DMD Global Database: Analysis of more than 7,000 Duchenne muscular dystrophy mutations. Hum Mutat 2015;36(4):395-402. DOI:10.1002 humu. 22758

22. Pellegrini C, Zulian A, Gualandi F, et al. Melanocytes-A novel tool to study mitochondria dysfunction in Duchenne muscular dystrophy. J Cell Physiol 2013;228(6):1323-1331. DOI:10.1002/ jср. 24290

23. Donald KAM, Mathema H, Thomas KGF, Wilmshurst JM. Intellectual and behavioral functioning in a South african cohort of boys with duchenne muscular dystrophy. J Child Neurol 2011;26(8):963-969. DOI:10.1177/0883073811399149 